\title{
Microempresarias en la comercialización de langosta en el mercado municipal de Bilwi
}

\author{
Micro-entrepreneurs women in the lobster commercialization in Bilwi's Municipal Market
}

\author{
Armando José Espinoza Padilla' \\ Mary Lau Williams²
}

\section{Resumen}

Esta investigación se realizó con el propósito de analizar los procesos de comercialización que realizan las microempresarias de langosta en el mercado municipal de Bilwi. El estudio es tipo mixto (cuantitativo y cualitativo) de un nivel descriptivo, debido a que los datos se cuantificaron para luego ser analizados según las variables formuladas y de acuerdo con el marco teórico descrito. La investigación es retrospectiva y de corte transversal, debido al estudio de la prevalencia de la exposición y del efecto en una muestra poblacional en un solo momento temporal. La información se obtuvo mediante encuestas y revisión documental. Los resultados relevantes han mostrado que las microempresarias del mercado municipal de Bilwi realizan la comercialización de langostas de forma empírica; proveen el producto de diferentes fuentes entre ellos pescadores artesanales, pequineras, acopiadores independientes que llegan desde las comunidades de los litorales o de las empresas pesqueras. También este estudio aporta información para que las autoridades del gobierno regional de la RACCN, el gobierno municipal de Puerto Cabezas, del Ministerio de Economía Familiar, Cooperativa y Comunitaria, así como entidades financieras diseñen e implementen políticas y estrategias de apoyo a este sector.

Palabras Clave: Comercialización, pequineras, pescador artesanal, mercado municipal, mujeres indígenas.

\section{Abstract}

This research was carried out with the purpose of analyzing the marketing processes carried out by lobster micro-entrepreneurs women in Bilwi Municipal Market. The study is a mixed type (quantitative and qualitative) of a descriptive level, because the data was quantified and then analyzed according to the variables formulated and along with the theoretical framework described. The research is retrospective and cross-sectional, due to the study of the prevalence of exposure and the effect on a population sample in a single temporal moment. The information was obtained through surveys and documentary review. The relevant results have shown that the micro entrepreneurs' women at Bilwi municipal market carry out the commercialization of lobsters empirically; they provide the product from different sources, among them artisanal fishermen, pequineras (women who are dedicated to buying and selling lobster), independent gatherers that arrive from coastal communities or fishing companies. This study also provides information for the authorities of the regional government of the RACCN, Puerto Cabezas municipal government, the Family, Cooperative and Community Economy Ministry, as well as financial entities to design and implement policies and strategies to support this sector.

Keywords: Marketing, pequineras, artisanal fisherman, municipal market, indigenous women.

\footnotetext{
1 Licenciado en Administración de Empresas con mención en Mercadotecnia por la Universidad de las Regiones Autónomas de la Costa Caribe Nicaragüense. Correo: armyespi@gmail.com ORCID: https://orcid.org/0000-0002-7230-413X,

2 Máster en Docencia Universitaria. Profesor de la Universidad de las Regiones Autónomas de la Costa Caribe Nicaragüense-Recinto Universitario Bilwi. Correo: mlauwilliams@gmail.com ORCID: https://orcid.org/0000-0001-7667-7745
}

Recibido: 25/06/2018 - Aprobado: 24/01/2019 


\section{Introducción}

La actividad pesquera en la Región Autónoma del Atlántico Norte de Nicaragua es una actividad ancestral que sirve actualmente como fuente de empleo, ingreso económico para el sostén del hogar, mejorar la dieta alimenticia de muchas familias, especialmente de los litorales norte y sur de la Costa Caribe Norte.

Actualmente, la pobreza, la falta de oportunidades de empleo, de apoyo del gobierno municipal, regional y nacional, obliga a las mujeres indígenas en la Región del Atlántico Norte del país a buscar nuevas formas de sobrevivencia, una de ellas es la comercialización de diversos productos en los diferentes mercados de la ciudad de Bilwi. Esta investigación se enmarcó en el análisis del proceso de comercialización de langosta común del Caribe (Panulirus argus), por mujeres indígenas en su mayoría miskitus, quienes funcionan como microempresarias en el mercado municipal de la ciudad de Bilwi, consideran que este recurso tiene demanda y un alto valor comercial en los mercados locales, nacionales e internacionales.

El tipo de estudio es cualitativo con elementos cuantitativo, descriptivo, retrospectivo y de corte transversal. En cuanto a su utilidad científica, los conocimientos obtenidos desde el punto de vista teórico y empírico, así como el análisis de los datos aportados en la investigación documental y de campo, aclararon lo referente a los procesos de comercialización de dicho recurso por microempresarias del mercado municipal.

En cuanto a su utilidad metodológica, podrá servir como herramienta confiable de tipo estadístico y cualitativo, las cuales podrán ser utilizadas como fuente de información a investigadores en la creación de instrumentos para el análisis y el manejo de información en el estudio de temas de carácter socioeconómico. De igual manera, podrá ser útil a futuros investigadores en el desarrollo de investigaciones relacionadas con el tema, y por último, debido a que es una investigación orientada al estudio de los procesos de comercialización de langostas, tiene muchas implicaciones técnicas, científicas y económicas por los múltiples problemas que enfrentan los seres humanos, las empresas y la sociedad en general.

En cuanto a la utilidad social, queda claramente establecida la necesidad de impulsar procesos de desarrollo económico, desde una perspectiva municipal con visión regional que permita a las personas beneficiarias sentirse satisfechas. En este sentido, los resultados de la presente investigación ayudarán a la toma de decisiones oportunas, para corregir sesgos y los rezagos de pobreza y diseñar planes, programas y proyectos orientados a mejorar la calidad de vida y el buen vivir de la familia costeña.

Finalmente, los resultados de esta investigación podrán servir como fuente de información y referencia bibliográfica para las personas naturales o jurídicas, estudiantes, docentes e investigadores de la Universidad de las Regiones Autónomas de la Costa Caribe Nicaragüense.

\section{Revisión de literatura}

La comercialización de productos del mar y en especial de la langosta común del Caribe (Panulirus argus) es una actividad realizada especialmente por los pueblos indígenas y afrodescendientes de los litorales del Caribe Norte y Sur de Nicaragua, como medio de subsistencia alimentaria e ingreso económico.

En este sentido, un estudio realizado en el 2007 por Trejos \& Valencia cuyo objetivo fue crear una empresa dedicada al procesamiento y comercialización de pescado ahumado y langosta, mostró que hay un nicho de mercado dispuesto a consumir estos productos. De igual forma Flores (2010), estudió a través de una caracterización, los hábitos y tendencias de consumo de pescados y mariscos, cuyo resultado reflejó que estos productos tienen una alta demanda. Sin embargo, en el estudio realizado por Bushey \& Rodas Matamoros y Reyes (2014) cuyo objetivo fue analizar las políticas de crédito hacia microempresarias del camarón, los resultados mostraron que las oportunidades de acceso al crédito de forma individual son limitadas. 
Seguidamente se hará un esbozo acerca de las teorías más importantes que soportan la investigación.

Proceso de comercialización. La comercialización es un proceso, que expresa cuatro aspectos. En el primero (cuándo), el autor se refiere al momento preciso de llevarlo a efecto; en el segundo aspecto (dónde), a la estrategia geográfica; el tercero (a quién), a la definición del público objetivo y finalmente (cómo), se hace una referencia a la estrategia a seguir para la introducción del producto en el mercado. Por lo tanto, están encaminados a planificar, fijar precios, promover y distribuir productos y servicios para satisfacer las necesidades de los consumidores; analizan y estudian las oportunidades de mercado, pero también en definir un plan de actuación dirigido a establecer los medios necesarios para que sus oportunidades se traduzcan en el cumplimiento de sus objetivos comerciales.

Mercado. Según Samuelson y Northaus (1999), es el conjunto de personas y organizaciones que participan en la compra y venta de bienes y servicios o en la utilización de esta; determinando precio y cantidad, pero en el que también confluyen circunstancias geográficas, sociales (hábitos), económicas (presión ejercida por la competencia), jurídicas (leyes vigentes), por lo tanto, el conocimiento de estas constituye un elemento básico para llevar a cabo esta operación. El mercado puede ser internacional, nacional, regional, local o municipal. Este último se refiere a establecimientos minoristas independientes, fundamentalmente de alimentación perecedera, agrupados en un edificio de titularidad pública y normalmente de uso exclusivo, que tienen servicios comunes y requieren una gestión de funcionamiento también común, según las fórmulas jurídicas establecidas en la legislación de régimen local, termino al cual hace referencia esta investigación.

En este sentido, el acto de mercadeo tiene como fin principal determinar las necesidades y deseos de los consumidores y proporcionar satisfacción deseada en forma más efectiva y eficiente que los consumidores, ya que es importante reconocer que las mayorías de las empresas se orientan a la producción y no el consumidor.

Mujeres microempresarias. De acuerdo con Chamorro (1998), es el conjunto de mujeres que trabajan independientemente en pequeños negocios, con escasos recursos y donde desarrollan un sin número de actividades en pequeña escala, con mano de obra familiar, especialmente en comercio, pulperías, panaderías, venta de ropa usada, mariscos, alimentos y servicios para producir, lo que resulta en procesos productivos de baja productividad y rentabilidad (Chamorro, 1998).

Pikineras. Son un grupo de mujeres miskitus, que se han organizado para dedicarse al acopio de mariscos que ni las grandes compañías desean. Entregan por adelantado el dinero a los buzos, y a cambio ellos les entregan las langostas, que luego revenden en el mercado de Puerto Cabezas. El vocablo proviene de "pikins" la parte de la captura de langosta que los empresarios rechazan y dejan que los buzos retengan, estos a su vez se las venden a las mujeres mariscadoras (Lacayo y Ergedas, 2013)

\section{Oferta y demanda}

De acuerdo con Rosenberg (1996), la oferta es la cantidad de bienes y servicios disponibles en un mercado a un determinado precio y en ciertas condiciones, por el que una persona está dispuesta a vender o comprar un determinado producto. La oferta puede clasificarse (Kottler y Armstrong, 2003) de acuerdo con el control que productores tienen con relación al mercado (oferta de mercado libre, oligopólica, y monopólica). Por otro lado, la demanda, de acuerdo con Krugman, Wellas \& Olney (2008) se refiere al deseo de comprar un bien y servicio en un momento concreto. Esta demanda puede ser elástica (significa que la variación porcentual de la cantidad demandada es mayor que la variación porcentual del precio), inelástica (cuando baja el precio, disminuye el ingreso total y cuando sube el precio, aumenta el ingreso total) o perfectamente inelástica (significa que no varía la cantidad demanda cuando el precio sube o baja)

Costos. De acuerdo con Greco y Godoy (2003), el costo es el valor del conjunto de bienes y esfuerzos en que se ha incurrido o incurrirá para obtener un producto terminado en condiciones de ser entregado al 
sector comercial para su comercialización, y que se mide en dinero mediante la reducción de activos o al incurrir en pasivos en el momento que se obtienen los bienes (Reyes, 1996).

Los costos pueden ser fijos y variables. Los costos fijos son irrecuperables, deben pagarse incluso cuando la empresa no produzca y no afecta la variación de la producción (ejemplo arrendamiento, sueldos, impuestos, entre otros). En cambio, los costos variables varían con el nivel de producción (la materia prima necesaria para producir, los obreros para cubrir las líneas de producción y la energía para poner en funcionamiento la empresa, etc.).

Pero los costos también pueden ser directos e indirectos. Los costos directos se asignan directamente a una unidad de producción y por lo general se asimilan a los costos variables; en cambio los indirectos son costos que se distribuye entre las diversas unidades productivas mediante algún criterio de reparto y en la mayoría de los casos son costos fijos.

Beneficios. Según la Real Academia Española (1992) se refiere a la utilidad o bien que se hace o recibe. Comercialmente se refiere a la ganancia económica que se obtiene de un negocio o inversión; y, en el sentido empresarial o contable a la renta, rendimiento, o provecho que se obtiene mediante la diferencia entre los ingresos derivados de las ventas y el costo total de la oportunidad de los recursos utilizados para producir los bienes o servicios. En este sentido podemos indicar que es un indicador de la creación de riqueza_o generación de valor_en la economía de una nación, y en el campo social como aquellas prestaciones otorgadas voluntariamente por el empleador, pero también el grado de satisfacción al adquirir un producto o servicio a un precio determinado.

Relación costo - beneficio. Indica la razón del retorno en dinero obtenido por cada unidad monetaria invertida y que esta relación se calcula dividiendo el ingreso bruto entre el costo total y si el cociente resulta igual a uno está en punto de equilibrio es decir no se pierde ni se gana, si resulta mayor que uno es rentable, pero si es menor que uno indica pérdidas. (Herrera, Velasco \& Radulavich, 1994).Para efectos de esta investigación la relación costo/ beneficio estará dirigida al análisis del costo que incurren las microempresarias para realizar la actividad económica y el beneficio económico obtenido por la inversión realizada, conocido como ganancia o utilidad.

Estrategias de comercialización. Según Sánchez (2015), son acciones que se llevan a cabo a fin de incrementar la eficiencia en los procesos de comercialización. Sigue expresando el autor que la parte sencilla de vender es pensar que alguien necesita del producto o servicio que se oferta y que está dispuesto a comprarlo, en caso contrario la empresa que no vende se extingue. La parte complicada es elaborar una estrategia para vender y en ella todos los integrantes de la organización deben estar enfocados en esta tarea, no sólo la parte comercial.

\section{Materiales y métodos}

El estudio tuvo un enfoque cualitativo con elementos cuantitativos, es descriptiva, retrospectiva, de corte transversal y como universo se tomaron en cuenta 26 personas: 6 microempresarias que venden langostas en el mercado municipal de Bilwi, y 20 clientes que compran langosta en el mercado. Sampiere (2006), define el universo como el conjunto de unidades de análisis para los cuales serán válidos los resultados y conclusiones de nuestro trabajo, constituyen el universo de estudio.

La muestra equivale al 100\% del universo y es cuando extraemos de la población un número menor de sujetos que tengan las mismas características de la población, a ese número menor de sujetos se le llama muestra. (Sampiere, Collado \& Lucio, 2006). 
Los datos primarios se obtuvieron de forma directa de los grupos de análisis (mujeres microempresarias vendedoras de langostas y clientes), a través de entrevista y encuesta; los datos secundarios fueron consulta a bibliografía digital, impresa, revistas y folletos relacionados al tema de investigación e internet.

En este sentido, Méndez (2008), explica que, para las fuentes secundarias, toda investigación implica acudir a este tipo de fuentes, que suministran información básica. Se encuentran en las bibliotecas y está contenida en libros, periódicos, trabajos de grado, revista especializadas, enciclopedia, diccionarios y páginas web de internet.

La información cuantitativa se presenta en tablas estadísticas y para el análisis se utilizó la estadística descriptiva. Y el programa SPSS y la información cualitativa se presentó en tablas matriciales y el análisis de la información se hizo encontrando diferencias y similitudes de los resultados encontrados según los objetivos específicos de la investigación.

\section{Resultados y discusión}

En cuanto al proceso de comercialización de langosta que implementan las microempresarias, el 100\% manifestaron que los productos (langostas) los adquieren de distintas fuentes: pescadores artesanales, piquineras, acopiadores independientes que llegan de las comunidades de los litorales o de las empresas pesqueras, rechazos o productos clasificados como clase "C". Posteriormente, alquilan vehículos para trasladar los productos desde el punto de compra hasta el mercado municipal para su venta a los clientes. Los resultados de entrevistas y de encuestas a clientes coyunturales y fijos son similares porque expresaron que las microempresarias compran el producto de distintos proveedores y luego lo venden en el mercado obteniendo un margen de ganancia.

El proceso de comercialización de langostas que implementan las microempresarias está representado en la figura 4, el que se relaciona con lo descrito por Kotler, P. (1995), quien describe que el proceso de comercialización e incluye cuatro aspectos fundamentales: ¿cuándo?, ¿dónde?, ¿a quién? y ¿cómo? En el primero, el autor se refiere al momento preciso de llevarlo a efecto; el segundo aspecto, a la estrategia geográfica; el tercero, a la definición del público objetivo y finalmente, hace referencia a la estrategia a seguir para la introducción del producto en el mercado.

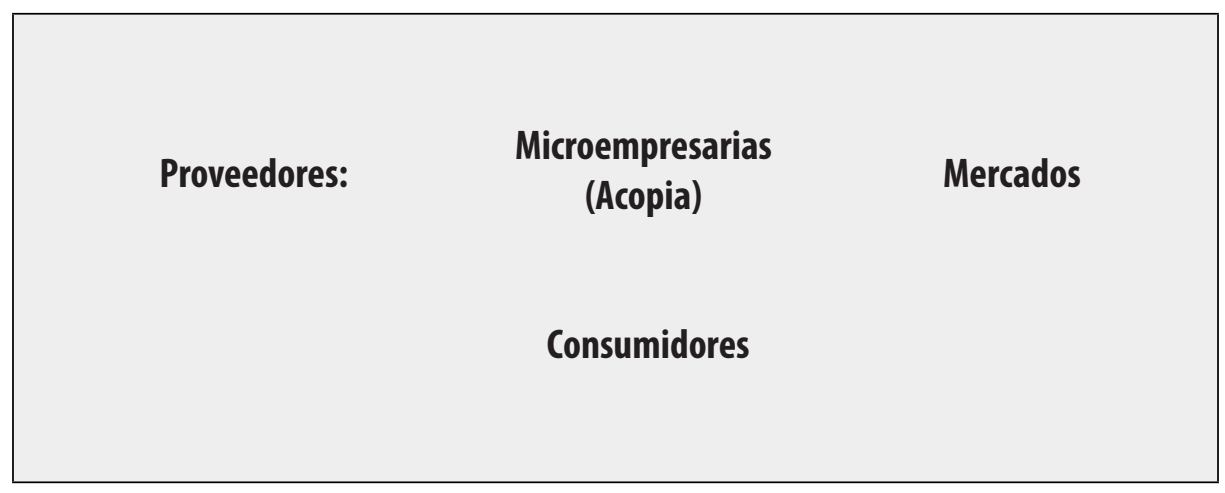

Figura 1: Flujograma del proceso de comercialización de langosta 
Los procesos de comercialización deben estar encaminados a planificar, fijar precios, promover, distribuir productos y servicios para satisfacer las necesidades de los consumidores, teniendo como objetivos fundamentales incrementar la cuota de mercado, la rentabilidad y el incremento de las ventas.

Relacionando, los resultados de instrumentos aplicados y las teorías descritas por diferentes autores sobre comercialización, se puede afirmar que las microempresarias del mercado municipal implementan procesos de comercialización de langostas de manera empírica o intuitiva, basadas en conocimientos ancestrales y experiencias de la vida.

Tabla 1: Oferta de langostas

\begin{tabular}{llllll}
\hline \multicolumn{1}{c}{$\mathrm{N}^{0}$} & \multicolumn{1}{c}{ OFERTA } & & MICROEMPRESARIAS & & $\%$ \\
\hline 01 & 5 a 10 & 1 & $10 \%$ & \\
02 & 40 a 50 Lbs & 1 & $23 \%$ & \\
03 & 100 a 150 Lbs & 2 & $33 \%$ & \\
04 & 200 a 300 Lbs & 1 & $17 \%$ & \\
05 & 300 a 500 Lbs & 1 & $17 \%$ & \\
\hline TOTAL & & 6 & $100 \%$ & \\
\hline
\end{tabular}

Fuente: Entrevista y encuestas, 2017

La relación oferta y demanda de langostas, es fluctuante, para clientes esporádicos, es de 5, 10, 15 libras y para clientes fijos de 40 hasta 500 libras. El período de oferta va de 3 a 5 días a la semana. Tabla- 1.

Tabla 2: Demanda de langosta

\begin{tabular}{lllll}
\hline No $^{\circ}$ & \multicolumn{1}{c}{ DEMANDA } & & MICROEMPRESARIAS & $\%$ \\
\hline 01 & 80 a 100 Lbs & 1 & $17 \%$ \\
02 & 200 a 300 Lbs & 1 & $17 \%$ \\
03 & 230 a 250Lbs & 1 & $17 \%$ \\
04 & Es variado & 1 & $17 \%$ \\
05 & Vendo lo que oferte & 2 & $32 \%$ \\
\hline TOTAL & & 6 & $100 \%$ \\
\hline
\end{tabular}

Fuente: Entrevista y encuestas-2017

La demanda de langostas de los clientes esporádicos es de 4 a 15 libras diarias y para clientes fijos oscila entre 80 a 300 libras diarias, aunque el $17 \%$ manifiesta que la demanda es variada y el $32 \%$ dice que vende la cantidad que oferte, ver tabla 2. El 100\% de las microempresarias que comercializan langostas en el mercado municipal de Bilwi, expresaron que venden la cantidad de langostas que ofertan porque tienen clientes que son dueños de comedores o restaurantes. Las microempresarias y clientes /consumidores, dijeron que la oferta de langostas satisface la demanda porque forma parte de la dieta alimenticia y se consume de diferentes maneras, especialmente en los comedores y restaurantes.

En este sentido se podría decir que la demanda es perfectamente inelástica ya que significa que no varía la cantidad demanda cuando el precio sube o baja coincidiendo con lo expresado por Krugman, Wells y Olney (2008) que la demanda se define como un bien y servicio que se desea comprar a un precio determinado en un momento concreto, relacionando lo que practican las microempresarias.

Rosenberg (1996), define la oferta como la cantidad de bienes y servicios disponibles en un mercado a un determinado precio, en ciertas condiciones, o bien, precio por el que una persona está dispuesta a 
vender o comprar un determinado producto, por tanto la oferta es la cantidad que está dispuesta a ofrecer un productor, si pudiera vender toda su producción y el propósito que se persigue es determinar o medir las cantidades, condiciones en que una economía puede y quiere poner a disposición del mercado un bien o servicio.

De acuerdo con los resultados de instrumentos aplicados a microempresarias, clientes y relacionado con lo descrito en el marco teórico, significa que la oferta es relativa o proporcional a la cantidad demanda de langostas. Esto implica que las microempresarias de manera empírica o en base a experiencias ancestrales, planifican la cantidad de productos que ofertan y que tienen conocimiento del mercado donde están posicionados y que satisface la demanda de sus clientes.

Los costos mensuales para la comercialización de langostas por microempresarias ubicadas en el mercado municipal fluctúan, según el origen de compra, transporte, insumos y materiales necesarios, la cantidad de compra y venta del producto.

Tabla 3. Costos mensuales para comercialización de langostas

\begin{tabular}{lll}
\hline$N^{0}$ & \multicolumn{1}{c}{ DESCRIPCION } & \multicolumn{1}{c}{ COSTOS ESTIMAD0 } \\
\hline 01 & Transporte Terrestre (Taxi) & \multicolumn{2}{c}{ CO $800.00-C \$ 1,000.00$} \\
02 & Transporte Acuático. & $C \$ 500.00$ \\
03 & Compra de bolsas & $C \$ 100.00-c \$ 300.00$ \\
04 & Hielo & $C \$ 160.00$ \\
05 & Soda & $C \$ 200.00$ \\
06 & Sal & $C \$ 160.00-c \$ 300.00$ \\
\hline
\end{tabular}

Fuente: Entrevistas y encuestas-2017

Greco y Godoy (2003) refieren que el costo es el "valor del conjunto de bienes y esfuerzos en que se ha incurrido o se va a incurrir para obtener un producto terminado en condiciones de ser entregado al sector comercial para su comercialización". Según este autor, el análisis de costos, se debería realizar incorporando todos los costos imputados por una unidad, es decir por una libra de langosta comprada y vendida para conocer con exactitud el costo unitario de comercialización, pero en este caso es difícil hacer los cálculos debido a que, la cantidad de compra y venta fluctúa, las microempresarias no llevan registros de ingresos y gastos, no planifican los costos, gastos sin incluir muchas actividades que generan costos y gastos. Es decir, en sus gastos o costos operacionales no incluyen todos los gastos y costos en los que incurren.

El precio de venta de langosta a sus clientes dependiendo del precio de adquisición del producto por libra. Si el precio de compra es $C \$ 70.00$ córdobas, el precio de venta es $C \$ 100.00$ y si se adquiere a $C \$$ 90.00 la venta a clientes es $C \$ 120.00$ córdobas por libra; es decir el margen de utilidades brutas es de $C \$$ 30.00 córdobas, lo que coincide con lo expresado por la mayoría de los clientes, aunque dos de los clientes dijeron que el margen de ganancia es menor $10 \%$.

Los precios de compra varían en dependencia de quien sea el proveedor del producto y oscila entre $C \$$ 80.00 y $C \$ 200.00$ córdobas, entonces las microempresarias para obtener ganancias duplican el precio de compra en relación con la venta, es decir las microempresarias no fijan precios basados en el costo, en el comprador o en la competencia. Ejemplo si compró a $C \$ 100.00$ la libra, el precio de venta es $C \$ 200.00$ córdobas. Otro elemento que incide en el precio de compra es el tamaño del producto que compran y venden. Ver tabla 4. 
Tabla 4: Precio de compra y venta de langosta

\begin{tabular}{|lll|}
\hline \multicolumn{1}{c}{ No } & \multicolumn{1}{c}{ COMPRA C\$ } & VENTA C\$ \\
\hline 01 & 70.00 & 100.00 \\
\hline & 100.00 & 120.00 \\
\hline 02 & 80.00 & 160.00 \\
\hline & 100.00 & 200.00 \\
\hline 03 & 70.00 & 100.00 \\
\hline & 80.00 & 110.00 \\
\hline 04 & 40.00 & 100.00 \\
\hline & 60.00 & 120.00 \\
\hline 05 & 60.00 & 120.00 \\
\hline 06 & 80.00 & 140.00 \\
\hline 07 & 70.00 & 140.00 \\
\hline Fuente: Entrevistas y encuestas-2017 & & \\
\hline
\end{tabular}

Según Valleta (1999), el beneficio es la ganancia, utilidad, renta, rendimiento, provecho que se obtiene mediante la diferencia entre los ingresos derivados de las ventas y el costo total de la oportunidad de los recursos utilizados para producir los bienes y en el sentido empresarial o contable se puede decir que es el exceso del ingreso por encima de todos los costes contractuales y de la amortización.

Entre los beneficios económicos que genera la comercialización de langostas a las microempresarias, es una ganancia mensual que oscila de $C \$ 2,000.00$ a $C \$ 3,500.00$ córdobas en temporadas altas. En cuanto a los beneficios sociales genera ingresos económicos para la familia, se logra pagar el estudio de los hijos, la energía eléctrica, agua, cable, adquisición de comida y vestimenta.

Si bien es cierto en el proceso de comercialización de langostas, las microempresarias obtienen utilidades brutas, pero si se hace un buen análisis incluyendo todos los costos y gastos que las microempresarias realizan, las ganancias o utilidades brutas son ficticias, mínimas o bien se encuentran en punto de equilibrio.

En relación con las alternativas para mejorar la eficiencia en los procesos de comercialización de langostas, se proponen:

1. Promover y fortalecer las capacidades comerciales y de servicios a las mujeres microempresarias vinculadas a la comercialización de langostas.

2. Facilitar el acceso a servicios de capacitación, asistencia técnica y financiamiento.

3. Fortalecer las formas propias de organización desarrolladas por las microempresarias que comercializan langostas en el mercado municipal.

4. Promover el intercambio de experiencias con microempresarias de otras regiones y departamento del país.

5. Identificar y promover formas innovadoras de comercialización de langostas en el mercado municipal.

6. Promover el desarrollo de nuevos productos con valor agregado. 
7. Desarrollar una cultura administrativa de los recursos económicos que se generan costos, fortalecimiento de conocimientos sobre comercialización y reinversión de los beneficios.

8. Buscar mecanismos para acercar oferta y demanda, que les permitan a las microempresarias obtener mejores precios por sus productos y a los consumidores precios más accesibles.

9. Promover alianzas estratégicas con las empresas públicas, privadas y proveedores de langostas.

Chain \& Chain (1997), describen las alternativas como los diferentes caminos que se pueden tomar para llegar a cumplir el objetivo propuesto, es decir modificar la situación actual en las condiciones, características y tiempo esperado lo que implica describir de forma general pero concreta, las acciones y Samuelson \& Nordhau (1998), definen eficiencia, como la ausencia de despilfarro o utilización de los recursos económicos que reporta el máximo nivel de satisfacción posible con los factores y la tecnología asignada.

La capacitación es considerada un tema esencial para qué las microempresarias puedan nutrirse de conocimientos de marketing a fin de lograr el éxito, los objetivos las metas, lo que podría dar como resultado maximización de las utilidades o ganancias en el retorno de la inversión, producto de la comercialización de langostas y minimización de los costos.

\section{v. Conclusiones}

De acuerdo con el análisis de los resultados y con base en los objetivos específicos de la presente investigación se llegó a las siguientes conclusiones:

Las mujeres microempresarias que comercializan langostas (Panulirus argus) en el mercado municipal de Bilwi, en su mayoría son de escasos recursos económicos, pero con mucha experiencia empírica en los negocios, la cual practican con el propósito de comercializar productos y servicios a la sociedad, generar recursos económicos líquidos para satisfacer sus necesidades básicas en una región donde la mayor parte de la familia está desempleada y prevalecen los mayores índices de pobreza.

El proceso de comercialización de langostas por microempresarias del mercado consiste en comprar el producto de diversos proveedores, entre ellos pescadores artesanales, piquineras, acopiadores independientes que llegan de las comunidades de los litorales o de las empresas pesqueras que luego trasladan a los mercados para ofertarlos a sus clientes.

La oferta del producto langosta es oligopólica, y la cantidad varía desde 40 hasta 500 libras, realizando dicha actividad entre 3 a 5 días a la semana y la cantidad de langosta que demandan los clientes esporádicos es un promedio de 15 libras diario y para clientes fijos oscila entre 60 y 300 libras diario.

Las microempresarias no han recibido ningún tipo de capacitación, por lo que hacen uso de sus conocimientos empíricos en las actividades de comercialización de langostas en el mercado municipal y las alternativas para promover la eficiencia en el proceso de la comercialización de la langosta son elementos que permitirán a las microempresarias tener otra visión acerca del marketing del producto.

\section{A manera de recomendaciones:}

\section{A microempresarias}

1. Realizar un mejor análisis del proceso de comercialización de langostas, incluyendo todos los costos y gastos en los que incurren, para qué las utilidades brutas y netas obtenidas sean reales y no ficticias o mínimas. 
2. Organizarse en cooperativas o asociaciones de mujeres microempresarias a través de un acta constitutiva con el fin de legalizar la actividad y facilitar gestiones de créditos en bancos, agencias financieras y otros.

\section{A la URACCAN}

1. Formular y desarrollar programas de capacitación a las microempresarias sobre procesos de comercialización de productos o políticas de marketing en coordinación con las PYMES y MEFCCA.

Al Gobierno Regional de la Costa Caribe Norte

1. Gestionar facilidades de créditos para este grupo de mujeres microempresarias a través de las PYMES y MEFCCA.

2. Desarrollar programas, proyectos y actividades enfocadas a mejorar las condiciones de acceso de las mujeres microempresarias a créditos, capacitación educación, información, redes sociales, etc.

\section{Lista de referencias}

Chain, S.N. \& Chain, S. R. (1997), preparación y Evaluación de proyectos, tercera edición. McGraw-Hill, Interamericana S.A, Santafe de Bogotá, D.C., Colombia.

Chamorro, D., (1998). El observador económico. FIDEG Managua. Nicaragua.

Greco, O., \& Godoy, A. (2003). Efectividad, economía, equidad, excelencia y Valoración de costos, $2^{\circ}$. Edición. Editorial Editorial Mc Graw Hill. Madrid.

Greco. O., (1999). Diccionario de economía, Valleta Ediciones, S.R.L, Ciudad de Buenos Aires, Argentina.

Herrera. F., Velasco, C., \& Radulovich, R., (1994). Fundamentos de análisis Económico. CATIE.

http://www.blogseitb.com/rogeblasco/2013/10/o7/rossana-lacayo-y-jose-ergedas-las-pikineras-de-los-cayos-miskitos-en-nicaragua/ Obtenido el 15 de abril, 2017.

https://www.entrepreneur.com/article/268753. Recuperado el 10 abril 2017.

Kotler (1995). Dirección de marketing. Análisis, planificación, gestión y Control. Tomo I y II. Editorial Mc Graw Hill, Madrid.

Krugman, P., Wells, R., \& Olney, M., (2008). Fundamentos de economía. Editorial Reverte, S.A. Barcelona España.

Lacayo R. y Ergedas J.(2013). Las pikineras de los cayos Miskitos en Nicaragua.

Lambin, J. (1995). Marketing Estrategico. 3 Edicion. Editorial Mc Graw Hill. Madrid.

Matamoros, B. y Reyes, Y (2007). Politicas de crédito de PANA PANA a mujeres microempresarias comercializadoras de camaron en el mercado municipal de Bilwi. (monografía)

Méndez, C.E., (2008). Fundamentos de metodología de Investigación. 4ta edición. México. 
Real Academia Española. Diccionario. (1992) 21ava Edicion. Editorial UNGRAF S.L Madrid.

Rosenberg J.M., (1996). Diccionario de Administración y Finanzas. Editorial, Océano, CENTRUM.S.A. Barcelona -España.

Sampieri, H.R., Fernández, C. C., \& Baptista, L.P., (2006). Metodología de la Investigación. 4ta edición. Editorial Mc Graw Hill. México.

Samuelson, P.A., \& Nordhaus, W.D., (1993). Economía. Decimoctava edición. Editorial McGraw Hill. España.

Samuelson, P.A., \& Nordhaus, W.D., (1998).Economía. Quintaedición. Editorial McGraw-Hill. Interamericana de España. S.A.U.

Sánchez H.G (2015). 7 estrategias para vender más. Artículo digital No.268756.

Trejos \& Valencia (20'07). Plan de negocios. Procesamiento y comercialización de pescado ahumado en el departamento de Risararalda. Universidad Tecnológica de Pereira. 